\title{
Giant vegetation of the tricuspid valve in a healthy patient without risk factors for right-sided endocarditis
}

rodolphe Durieux ${ }^{1}$, Valérie Henrard ${ }^{2}$, Renzo Granato ${ }^{1}$, Mérédith Knapen ${ }^{1}$, Philippe

Amabili $^{1}$, and Jean Olivier Defraigne ${ }^{1}$

${ }^{1}$ Centre hospitalier universitaire de Liege

${ }^{2}$ Groupe Sante CHC

January 18,2022

\begin{abstract}
We present the case of a middle-aged man who developed an infective endocarditis of the tricuspid valve caused by methicillinsensitive Staphylococcus aureus and associated with a very large vegetation (nearly $5 \mathrm{~cm}$ ). Besides the quite unusual size of the vegetation, this report highlights that severe right-sided endocarditis can occur in the absence of classical risk factors (intravenous drug abuse, presence of a cardiac implantable electronic device or other intravascular device, and underlying rightsided cardiac anomaly) and that some cases of severe tricuspid endocarditis can be successfully treated by partial excision and patch repair.
\end{abstract}

Giant vegetation of the tricuspid valve in a healthy patient without risk factors for right-sided endocarditis

Rodolphe Durieux $\mathrm{PHD}^{\mathrm{a}}$, Valérie Henrard $\mathrm{MD}^{\mathrm{b}}$, Renzo Granato $\mathrm{MD}^{\mathrm{a}}$, Mérédith Knapen $\mathrm{MD}^{\mathrm{c}}$, Philippe Amabili PHD ${ }^{\mathbf{c}}$, Jean-Olivier Defraigne PHD ${ }^{\mathrm{a}}$

a department of cardiothoracic surgery, University Hospital of Liège; Liège, Belgium

b department of cardiology, Notre-Dame Clinic; Hermalle-sous-Argenteau, Belgium

${ }^{\mathrm{c}}$ department of anesthesiology, University Hospital of Liège; Liège, Belgium

Running Head : Giant vegetation of the tricuspid valve

Keywords : Cardiovascular pathology

Corresponding author : Rodolphe Durieux

Department of cardiothoracic surgery, University Hospital of Liège; Domaine Universitaire du Sart-Tilman, B35 -4000 Liège, Belgium

Tel: 003243667163

Fax: 003243667410

rdurieux@chuliege.be

Funding : none

Abstract 
We present the case of a middle-aged man who developed an infective endocarditis of the tricuspid valve caused by methicillin-sensitive Staphylococcus aureus and associated with a very large vegetation (nearly 5 $\mathrm{cm}$ ). Besides the quite unusual size of the vegetation, this report highlights that severe right-sided endocarditis can occur in the absence of classical risk factors (intravenous drug abuse, presence of a cardiac implantable electronic device or other intravascular device, and underlying right-sided cardiac anomaly) and that some cases of severe tricuspid endocarditis can be successfully treated by partial excision and patch repair.

\section{Case report}

A 61-year-old man was admitted to hospital for chills, myalgia and anorexia evolving for two weeks. His past medical history was notable for hypertension, hypercholesterolemia, and tobacco abuse. Laboratory tests on admission showed an elevated C-reactive protein at $161 \mathrm{mg} / \mathrm{dl}$, a hyperleukocytosis at 17 000/mm3, elevated total and direct bilirubin levels and liver enzyme alteration. Thoraco-abdominal CT-scan revealed the presence of multiple bilateral pulmonary consolidations compatible with lung abscess. Repeat blood cultures were positive for a methicillin-sensitive Staphylococcus aureus (MSSA). Transesophageal echocardiogram (TEE) (Figure 1) showed the presence of a huge right ventricular mass attached to the anterior leaflet of the tricuspid valve associated with a moderate tricuspid regurgitation.

The most likely diagnosis was an infective tricuspid valve endocarditis caused by MSSA, with a large vegetation and complicated by pulmonary abscess. Intravenous antibiotic therapy with cefazoline (preferred to flucloxacillin in the context of impaired liver enzymes) was initiated and the patient was scheduled for an urgent surgery due to the risk of massive pulmonary embolism.

Surgery under median sternotomy was preferred to a minimally invasive approach due to the embolic risk associated with the Seldinger technique required for peripheral venous cannulation. At the opening of the right atrium, we discovered a giant lobulated mass attached to the anterior leaflet of the tricuspid valve by a broad pedicle, near the antero-septal commissure (Figure 2). The mass was removed, excising part of the anterior leaflet (Figure 3). The defect was repaired by a bovine pericardial patch and two artificial neochordae were passed to the free margin of the pericardial patch and attached to the anterior papillary muscle. The antero-septal commissure was closed by an edge-to-edge stich and an annuloplasty using a 34 mm Carpentier-Edwards Physio Tricuspid ring was performed.

Postoperative echocardiography demonstrated a good functional result on the tricuspid valve, with trace residual tricuspid regurgitation without stenosis (mean transvalvular gradient of $1.8 \mathrm{mmHg}$ ). The resected mass was composed of fibrin deposition on microscopic examination, consistent with an infectious vegetation. Culture of the vegetation revealed the presence of MSSA and intravenous cefazoline was continued for 42 days after surgery. The postoperative course was uneventful. The 3-month follow-up assessment was satisfactory, showing an excellent tricuspid valve function without signs of infection recurrence.

\section{Ethics statement}

Approval for publication was obtained from the patient and from our Institutional Review Board.

\section{Data availability statement}

The dataset on which this paper is based is available from the corresponding author upon reasonable request.

\section{Conflict of interest statement}

The authors have no conflict of interest to declare.

Legend Figure 1. Transesophageal echocardiogram (mid esophageal right ventricle inflow-outflow view) demonstrating a giant mobile mass (asterisk) attached to the anterior leaflet of the tricuspid valve. RA, right atrium; RV, right ventricle; Arrow, tricuspid valve.

Legend Figure 2. Intraoperative view of a giant polypoid-like mass attached to the anterior leaflet of the tricuspid valve, near the antero-septal commissure. AL, anterior leaflet; PL, posterior; SL; septal leaflet; Asterisk, vegetation; Arrow, antero-septal commissure. 
Legend Figure 3. Resected yellowish, polypoid vegetation greater than $45 \mathrm{~mm}$ in size, with a part of the anterior tricuspid leaflet
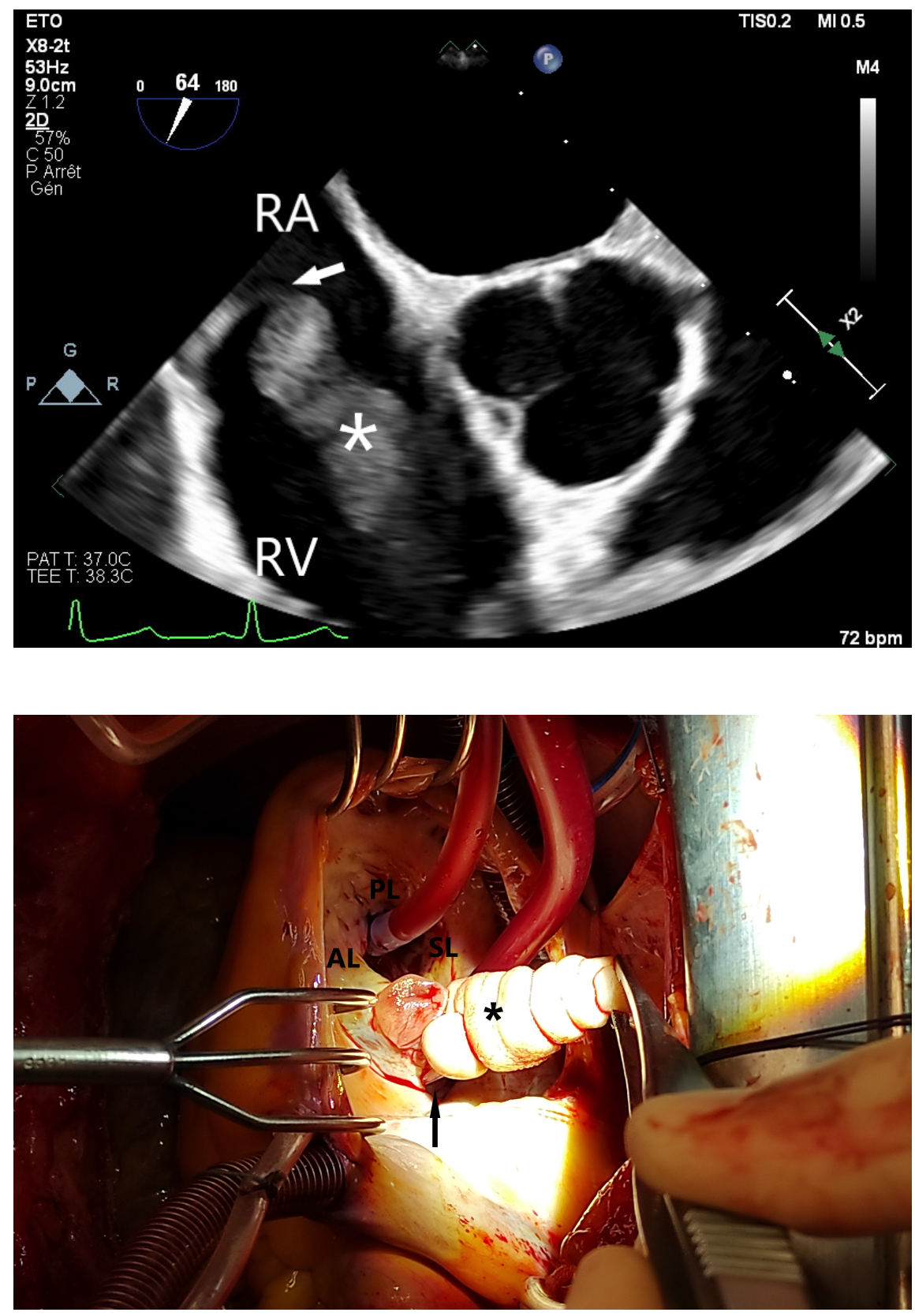


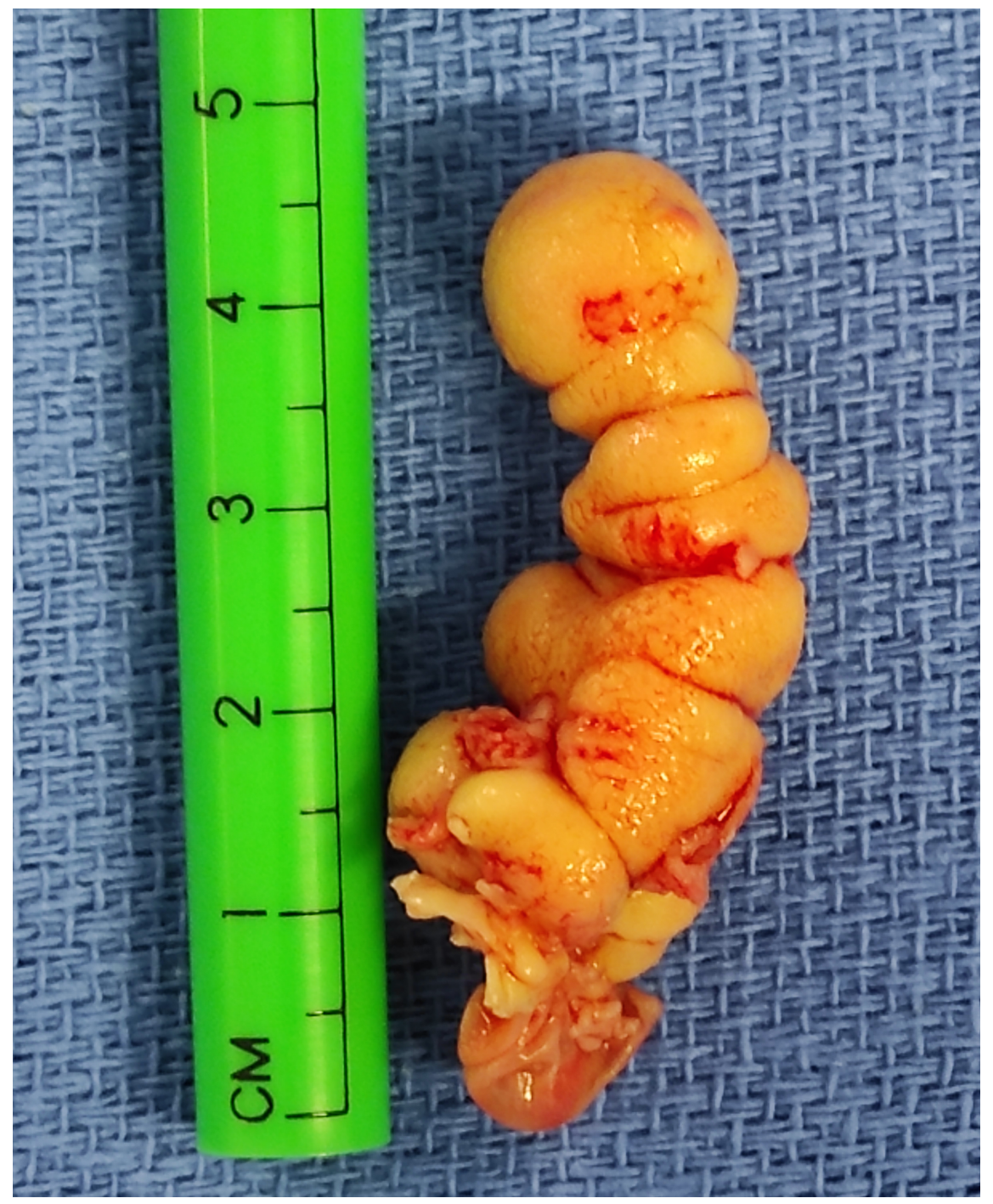

